\title{
Avaliação do estado cognitivo e capacidade funcional em pessoas idosas institucionalizadas
}

\author{
Assessment of cognitive status and functional capacity in institutionalized older adults \\ Evaluación del estado cognitivo y capacidad funcional en ancianos institucionalizados
}

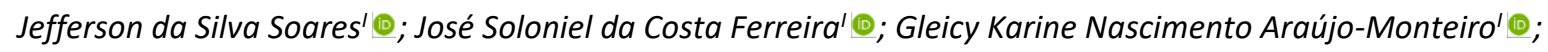 \\ Rafaella Queiroga Souto' @ ; João Euclides Fernandes Braga' $\bullet$
}

'Universidade Federal da Paraíba, João Pessoa, PB, Brasil

\begin{abstract}
RESUMO
Objetivo: analisar a relação entre o estado cognitivo, o nível de capacidade funcional e os fatores socioeconômicos entre pessoas idosas institucionalizadas. Métodos: estudo quantitativo, transversal, desenvolvido com pessoas idosas em Instituição de Longa Permanência para Idosos (ILPI). Os instrumentos utilizados: questionário Brazil Old Age Schedule; Mini exame do Estado Mental; escalas de Atividades Avançadas de Vida Diária, Atividades Instrumentais de Vida Diária e Atividades Básicas de Vida Diária. A análise foi realizada através de estatística descritiva e estatística inferencial. Resultados: predominou o déficit cognitivo entre pessoas idosas do sexo masculino $(50,0 \%)$, até 70 anos $(57,1 \%)$, alfabetizados $(58,6 \%)$, em um relacionamento $(66,7 \%)$, sem filhos $(60,5 \%)$ e com renda superior a um salário mínimo (66,7\%). A maioria das pessoas idosas com déficit tem menor nível nas atividades avançadas $(71,9 \%)$, independência nas atividades instrumentais $(55,6 \%)$ e dependência nas atividades básicas $(71,1 \%)$. Conclusão: o déficit cognitivo relaciona-se com a capacidade funcional, sendo menor em pessoas idosas com maior nível de independência e mais ativos.

Descritores: Idoso; Saúde do Idoso; Instituição de Longa Permanência para Idosos; Atividades Cotidianas.
\end{abstract}

\begin{abstract}
Objective: to analyze the relationship between cognitive status, level of functional capacity and socioeconomic factors among institutionalized older adults. Method: in this quantitative cross-sectional study of older adults in long-stay institutions for the elderly, the instruments used were the Brazil Old Age Schedule questionnaire, the Mini Mental State Exam, and the Advanced Activities of Daily Living, Instrumental Activities of Daily Living, and Basic Activities of Daily Living scales. The data were analyzed using descriptive and inferential statistics. Results: cognitive deficit predominated among participants who were elderly males (50.0\%), up to 70 years old $(57.1 \%)$, literate $(58.6 \%)$, in a relationship $(66.7 \%)$, childless $(60.5 \%)$ and with income of more than one minimum wage $(66.7 \%)$. Most older adults with deficits scored lower in advanced activities $(71.9 \%)$, independence in instrumental activities (55.6\%) and dependence in basic activities (71.1\%). Conclusion: cognitive deficit is related to functional capacity, and is lower in more active elderly people with higher levels of independence.

Descriptors: Aged; Health of Elderly; Homes for the Aged; Activities of Daily Living.
\end{abstract}

\section{RESUMEN}

Objetivo: analizar la relación entre estado cognitivo, nivel de capacidad funcional y factores socioeconómicos entre ancianos institucionalizados. Métodos: estudio cuantitativo, transversal, desarrollado junto a ancianos en Instituciones de Larga Estancia para Ancianos (ILPI). Los instrumentos utilizados: Cuestionario Brazil Old Age Schedule; Mini examen del estado mental; escalas de Actividades Avanzadas de la Vida Diaria, Actividades Instrumentales de la Vida Diaria y Actividades Básicas de la Vida Diaria. El análisis se realizó mediante estadística descriptiva y estadística inferencial. Resultados: Predominó el déficit cognitivo entre los hombres ancianos $(50,0 \%)$, hasta los 70 años $(57,1 \%)$, alfabetizados $(58,6 \%)$, que están en pareja $(66,7 \%)$, sin hijos $(60,5 \%)$ y con ingresos superiores a un salario mínimo $(66,7 \%)$. La mayoría de los ancianos con déficit tiene un menor nivel en actividades avanzadas $(71,9 \%)$, independencia en actividades instrumentales $(55,6 \%)$ y dependencia en actividades básicas $(71,1 \%)$. Conclusión: el déficit cognitivo está relacionado con la capacidad funcional, siendo más bajo en ancianos con mayor nivel de independencia y más activos.

Descriptores: Anciano; Salud del Anciano; Hogares Para Ancianos; Actividades Cotidianas.

\section{INTRODUÇÃO}

O processo de envelhecer acomete cada indivíduo de forma singular e pode trazer alterações na vida das pessoas idosas. As transformações morfológicas, funcionais e bioquímicas são inerentes ao processo de envelhecer e influenciam diretamente na capacidade de realizar atividades do cotidiano. 0 decréscimo da capacidade neuromotora acarreta a perda progressiva da autonomia, aumenta o nível de dependência e, assim, a vulnerabilidade ${ }^{1}$.

A cognição é uma função psicológica de aquisição de conhecimento, por meio de processos como a percepção de estímulos, o raciocínio e a formação de respostas a estímulos externos. A perda da cognição é, principalmente, efeito 
do comprometimento do sistema nervoso central que ocorre com o avançar da idade. Esse processo é gradual, e tem maior incidência nas pessoas acima de 80 anos $^{2}$.

A perda de funções cognitivas é consequência do processo de envelhecer e podem ser potencializadas por problemas de saúde. Assim, o modo que se envelhece é determinante para a progressão do estado cognitivo. Outro efeito do déficit cognitivo é o declínio funcional, assim, prejudicando a capacidade de desenvolver atividades do cotidiano e, por consequência, diminuindo a autonomia ${ }^{3}$.

O declínio da capacidade funcional está relacionado à redução do poder de decidir e atuar nas atividades diárias. O déficit dessa aptidão refere-se à falta de autonomia e independência para poder gerir a própria vida, por conseguinte, a incapacidade de autocuidado. O aumento da dependência da pessoa idosa o torna mais vulnerável ao abuso físico, psicológico ou financeiro ${ }^{4}$.

A diminuição do estado cognitivo e da capacidade funcional são processos que acarretam, diretamente, na falta de autonomia e independência. Assim, a inabilidade para atividades cotidianas aumenta a necessidade de um acompanhamento da família ou de um profissional. $O$ estresse causado entre os familiares e a ausência de condições para os cuidados em domicílio são, por vezes, o motivo de institucionalização ${ }^{5}$.

O encaminhamento de pessoas idosas para as ILPIs se dá em condições de necessidade de acompanhamento profissional. Essas entidades são residências coletivas que atendem pessoas idosas com ou sem suporte familiar, bem como aqueles com dificuldades para o exercício das atividades diárias, que precisam de cuidados prolongados ${ }^{4}$.

A adaptação das pessoas idosas nas ILPIs é um processo repleto de alterações não só ambientais, mas de caráter social. A mudança na posição familiar do sujeito, além da realocação abrupta promove uma situação de vulnerabilidade e estresse psicológico ao sujeito. Estas mudanças do cotidiano e inserção de novos hábitos acentuam o comprometimento cognitivo ${ }^{6}$

A realização da presente pesquisa justifica-se pela necessidade de identificar e descrever quais fatores estão relacionados ao déficit cognitivo e à capacidade funcional nas pessoas idosas que residem em ILPIs. Diante deste contexto, a pesquisa teve por objetivo analisar a relação entre o estado cognitivo, o nível de capacidade funcional e os fatores socioeconômicos entre pessoas idosas institucionalizadas.

\section{REVISÃO DA LITERATURA}

O envelhecimento da população é um processo que ocorre a nível mundial, mas não é considerado um efeito recente, haja vista que países como Portugal e França convivem com as consequências desse processo há várias décadas. Pode ser considerado relativo, porém, a Organização Mundial de Saúde (OMS) considera, em países em desenvolvimento, que a pessoa idosa é aquela com idade a partir de 60 anos ${ }^{1,3}$.

Esse processo é caracterizado por declínio fisiológico, portanto, tem como consequência a diminuição da capacidade funcional, déficit cognitivo e aparecimento de doenças crônicas. Esses efeitos do envelhecimento têm como resultado a dependência nas Atividades de Vida Diárias (AVD) e as funções envolvidas no processo cognitivo, como o aprendizado e a memória ${ }^{3,5}$. Assim, a manutenção da independência nas AVD e da capacidade cognitiva, não está relacionada apenas na prevenção de doenças, mas também na possibilidade de bem-estar e qualidade de vida das pessoas idosas. A continuidade da capacidade funcional depende da habilidade do indivíduo em se adaptar aos desafios físicos, mentais e sociais que, inevitavelmente, ocorrem ao longo da vida, sendo que isso, do mesmo modo, ocorre durante a institucionalização ${ }^{4-6}$.

O estudo da capacidade cognitiva e funcional em ILPIs representa a base para promoção da própria saúde e de gestão do ambiente domiciliar por parte das pessoas idosas, levando em consideração a escassez de pesquisas na área, salientando que o déficit dessas áreas representa risco de violência, dependência, mortalidade e hospitalização ${ }^{4-6}$.

\section{MÉTODO}

Tratou-se de um estudo quantitativo, descritivo, do tipo transversal, guiado pela ferramenta Strengthening the Reporting of Observational Studies in Epidemiology (STROBE). Foi realizado em duas instituições filantrópicas, localizadas na cidade de João Pessoa-PB, durante o período de 2018 a 2019.

Foram incluídos todos os indivíduos com 60 anos ou mais, residentes nas instituições do estudo. Os que não apresentaram condições de responder aos questionamentos, participaram com o apoiador ou cuidador responsável. Integraram a amostra do estudo 90 pessoas idosas.

Foi realizado o treinamento dos pesquisadores para a realização adequada da coleta de dados, responsável e imparcial junto aos participantes da pesquisa. Nessa ocasião, a pesquisa, seus objetivos, os instrumentos para coleta de 
dados e o Termo de Consentimento Livre e Esclarecido (TCLE) foram apresentados. Durante a capacitação todas as dúvidas foram sanadas. Ao todo, o treinamento teve duração de oito horas.

A coleta de dados foi realizada no período entre agosto e outubro de 2018, nas ILPIs, em local reservado, livre de interrupções e interferências externas, onde a pessoa idosa esteve à vontade para responder aos questionários junto com os pesquisadores ou acompanhada do cuidador, que respondeu ao questionário referente à sua situação. A coleta de dados se deu após apresentação dos pesquisadores, esclarecimentos sobre os objetivos e o sigilo dos dados, da disponibilidade em participar e assinatura do TCLE.

Para a coleta de dados foram utilizados os instrumentos: questionário Brazil Old Age Schedule (BOAS), aplicando as perguntas correspondentes às condições socioeconômicas; ; o Mini Exame do Estado Mental (MEEM), para avaliação cognitiva $^{8}$; as escalas de Atividades Instrumentais de Vida Diária (AIVD) ${ }^{9}$, Atividades Básicas de Vida Diária (ABVD) ${ }^{10}$ e as Atividades Avançadas de Vida Diária $(A A V D)^{11}$, mensurando a capacidade funcional.

A escala BOAS é definida com uma ferramenta de característica multidimensional com aplicabilidade para a população idosa, que tem nível de aceitabilidade alta e pode gerar informações como: aspectos físicos e mentais; mensurar nível econômico; identificar a situação social ${ }^{7}$. Tal escala foi utilizada com intenção de investigar situações relacionadas ao perfil social e econômico da pessoa idosa.

O MEEM é um teste breve de rastreio cognitivo para identificação de demência, composto por 30 itens. Os itens avaliados pelo MEEM são: orientação, memória imediata, atenção e cálculo, memória de evocação e linguagem ${ }^{8}$. Sua escolha foi baseada no histórico de uso por pesquisadores renomados em diferentes países. Permite avaliar o déficit cognitivo das pessoas idosas.

A avaliação da capacidade funcional da pessoa idosa foi realizada pelo uso de escalas. O índice de Katz para as $A B V D^{11}$ e a escala de Lawton e Brody para avaliação das AIVD ${ }^{9}$. A primeira diz respeito à autonomia diante de atividades ligadas ao autocuidado (comer, tomar banho, etc.); a segunda direciona-se às atividades que englobam maior liberdade em executar atividades (dirigir, realizar compras, ir ao banco, etc.).

A escala das AAVDs também faz parte da avaliação da capacidade funcional. É aplicada na intenção de mensurar a inserção da pessoa idosa em atividades de participação social, voluntárias, educacionais, ou seja, atividades que dependem do interesse da própria pessoa idosa ${ }^{12}$.

Os dados coletados foram tabulados e analisados no software SPSS, versão 26.0. A digitação dos dados se deu em dupla entrada, ou seja, duas digitações em diferentes planilhas por digitadores distintos, com intuito a diminuir erros de consistência e digitação. Após a conclusão da digitação, os dois bancos foram comparados com a finalidade de minimizar erros de digitação.

Os dados foram analisados por meio de estatística descritiva, utilizando a distribuição de frequência; e inferencial, por meio de testes de associação e de correlação. O nível de significância utilizado foi de $5 \%(p$-valor $<0,05)$ para todos os testes estatísticos utilizados. Foram utilizados os testes qui-quadrado de Pearson, Exato de Fisher e de Correlação de Pearson.

O teste paramétrico foi utilizado, devido ao resultado do Teste de Kolmogorov Smirnov apresentar uma distribuição com tendência à normalidade.

A pesquisa foi aprovada pelo Comitê de Ética em Pesquisa da instituição, atendendo aos preceitos éticos de pesquisas com seres humanos.

\section{RESULTADOS}

A Tabela 1 exibe os dados sobre da associação dos dados sociodemográficos com o déficit cognitivo.

Observa-se o predomínio entre pessoas idosas do sexo masculino (50,0\%; n=12), com 60 a 70 anos $(57,1 \%$; $n=8)$, alfabetizados $(58,6 \% ; n=34)$, com relacionamento $(66,7 \% ; n=4)$, sem filhos $(60,5 \% ; n=23)$ e que recebem mais de um salário mínimo $(66,7 \% ; n=6)$.

A Tabela 2 demonstra a associação da capacidade funcional e o déficit cognitivo.

Foi possível identificar que as pessoas idosas com déficit, em sua maioria, foram as menos ativas e com maior dificuldade nas atividades avançadas (71,9\%; $n=23)$, independentes nas atividades instrumentais $(55,6 \% ; n=5)$ e dependente nas atividades básicas $(71,1 \% ; n=27)$. Verifica-se associação significativa, sob o ponto de vista estatístico, entre o déficit cognitivo e as atividades avançadas e básicas.

$\mathrm{Na}$ Tabela 3, verifica-se a correlação entre as variáveis de escore de atividades físicas e o escore do MEEM.

Identificou-se que todas as variáveis apresentam correlação significativa, sob o ponto de vista estatístico, com o déficit cognitivo. No escore de atividades avançadas teve uma correlação positiva e moderada $(0,488)$, e nas atividades instrumentais e básicas uma correlação negativa e moderada $(-0,583)$ e $(-0,503)$, respectivamente. 
TABELA 1: Associação entre as variáveis sociodemográficas com o déficit cognitivo entre os indivíduos participantes. João Pessoa, PB, Brasil, 2018

\begin{tabular}{|c|c|c|c|}
\hline \multirow[b]{2}{*}{ Variáveis } & \multicolumn{2}{|c|}{ MEEM } & \multirow[b]{2}{*}{ p-valor } \\
\hline & $\begin{array}{l}\text { Com déficit } \\
\text { n (\%) }\end{array}$ & $\begin{array}{l}\text { Sem Déficit } \\
\text { n (\%) }\end{array}$ & \\
\hline \multicolumn{4}{|l|}{ Sexo } \\
\hline Masculino & $12(50,0)$ & $12(50,0)$ & $0,437^{*}$ \\
\hline Feminino & $24(40,7)$ & $35(59,3)$ & \\
\hline \multicolumn{4}{|l|}{ Idade } \\
\hline 60 a 70 anos & $8(57,1)$ & $6(42,9)$ & $0,966^{*}$ \\
\hline$>70$ anos & $39(56,5)$ & $30(43,5)$ & \\
\hline \multicolumn{4}{|l|}{ Escolaridade } \\
\hline Alfabetizado & $34(58,6)$ & $24(41,4)$ & $0,474^{*}$ \\
\hline Analfabeto & $12(50,0)$ & $12(50,0)$ & \\
\hline \multicolumn{4}{|l|}{ Estado conjugal } \\
\hline Casado/morando junto & $4(66,7)$ & $2(33,3)$ & $0,693 * *$ \\
\hline Viúvo/divorciado/nunca se casou & $43(55,8)$ & $34(44,2)$ & \\
\hline \multicolumn{4}{|l|}{ Teve filhos } \\
\hline Sim & $24(53,3)$ & $21(46,7)$ & $0,510^{*}$ \\
\hline Não & $23(60,5)$ & $15(39,5)$ & \\
\hline \multicolumn{4}{|l|}{ Renda } \\
\hline Até 1 salário mínimo & $18(51,4)$ & $17(48,6)$ & $0,477 * *$ \\
\hline Mais de 1 salário & $6(66,7)$ & $3(33,3)$ & \\
\hline
\end{tabular}

Nota: * Teste de Qui-quadrado de Pearson; **Teste Exato de Fisher.

TABELA 2: Associação entre a capacidade funcional e o déficit cognitivo entre as pessoas idosas. João Pessoa, PB, Brasil, 2018

\begin{tabular}{llll}
\hline Variáveis & \multicolumn{2}{c}{ MEEM } & \\
& \multicolumn{1}{c}{$\mathbf{n}(\%)$} & $\begin{array}{c}\text { Com déficit } \\
\mathbf{n}(\%)\end{array}$ & Sem Déficit \\
\hline $\begin{array}{l}\text { Atividades avançadas } \\
\text { Mais ativo }\end{array}$ & $22(44,9)$ & $27(55,1)$ & $0,017^{*}$ \\
$\begin{array}{l}\text { Menos ativo } \\
\text { Atividades instrumentais } \\
\text { Independente }\end{array}$ & $23(71,9)$ & $9(28,1)$ & \\
$\begin{array}{l}\text { Dependente } \\
\text { Atividades básicas }\end{array}$ & $5(55,6)$ & $4(44,4)$ & $0,628^{* *}$ \\
$\begin{array}{l}\text { Independente } \\
\text { Dependente }\end{array}$ & $16(54,9)$ & $32(45,1)$ & \\
\hline
\end{tabular}

Nota: * Teste de Qui-quadrado de Pearson; **Teste Exato de Fisher.

TABELA 3: Correlação entre o escore total do MEEM e as atividades avançadas, instrumentais e básicas da vida diária. João Pessoa, PB, Brasil, 2018

\begin{tabular}{lcc}
\hline \multirow{2}{*}{ Variáveis } & \multicolumn{2}{c}{ Escore total do MEEM } \\
& Coeficiente de correlação & p-valor* \\
\hline Escore atividades avançadas & 0,488 & $<0,001$ \\
Escore atividades instrumentais & $-0,583$ & $<0,001$ \\
Escore atividades básicas & $-0,503$ & $<0,001$ \\
\hline
\end{tabular}

Nota: *Teste de Correlação de Pearson

\section{DISCUSSÃO}

Os dados do presente estudo apontam que o número de mulheres foi superior ao de homens nas ILPIs, corroborando com os achados apresentados em outros estudos ${ }^{13,14}$. No entanto, um número menor de mulheres apresentou déficits cognitivos, podendo ser justificado pela maior preocupação com a saúde e com o autocuidado ${ }^{15}$. 
A predominância do déficit cognitivo entre idosas está em conformidade com outros estudos ${ }^{16}$, isso pode ser explicado pela maior longevidade feminina, dessa forma, prolongando a exposição a fatores dessa condição neuropsicológica. Outra explicação seria que, apesar de apresentarem maior expectativa de vida, as mulheres têm uma baixa qualidade de vida, deixando-as mais susceptíveis a essa condição ${ }^{17 .}$

Com relação à faixa etária, verificou-se uma predominância de déficit cognitivo em pessoas idosas com idade entre 60 e 70 anos, contrariando alguns estudos, que defendem que a idade mais avançada se configura como uma maior possibilidade de surgir comorbidades em pessoas idosas institucionalizadas ${ }^{2,15,18}$.

No tocante à escolaridade, viu-se que a maioria dos indivíduos alfabetizados apresentou maior déficit, quando comparado ao grupo sem alfabetização. Esse dado diverge do resultado apresentado em estudo de revisão, no qual, é revelado que quanto maior a escolaridade, menor é o déficit cognitivo ${ }^{6}$. Entretanto, é preciso levar em consideração que fatores biológicos podem afetar a cognição, independente da escolaridade do sujeito.

Ao observar o estado conjugal, notou-se a prevalência de pessoas idosas institucionalizadas sem companheiro. Os dados também destacam a prevalência de déficit cognitivo entre indivíduos sem filhos. A ausência de um companheiro e a solidão podem ser fatores desencadeadores de doenças associadas como a depressão ${ }^{19}$.

Quanto à renda, os resultados demonstram a prevalência do estado cognitivo com déficit em pessoas idosas com mais de um salário. Este achado difere do resultado de uma pesquisa realizada em um município do sul do Brasil, que associou baixa renda com maior probabilidade de declínio cognitivo ${ }^{17}$. Entretanto, é preciso levar em consideração que o estado cognitivo tem relação com a qualidade de vida e que esta não depende exclusivamente do poder de compra ${ }^{20}$

A partir da análise da associação entre capacidade funcional e déficit cognitivo, pôde-se notar que há um menor nível de ação para atividades avançadas entre as pessoas idosas com declínio cognitivo. As AAVD são atividades complexas que envolvem funcionamento físico, psicológico e social. A dificuldade no desenvolvimento dessas atividades aparece ao longo do envelhecimento e se acentuam na velhice, caracterizando declínio na capacidade cognitiva ${ }^{5}$.

As AIVD demandam do indivíduo funções que possibilitam a autonomia para atividades como preparar refeições, gerir o próprio dinheiro e lidar com transportes. Os dados do estudo demonstram a prevalência da independência nessas atividades por pessoas idosas com déficit cognitivo, divergindo com o estudo realizado em Pelotas ${ }^{21}$.

No que diz respeito às atividades básicas, percebeu-se que a maioria dos participantes da pesquisa com déficit cognitivo é dependente. Nesse quesito, a associação mostrou significância estatística. Os achados sugerem que o envelhecimento fisiológico acarreta o déficit da capacidade cognitiva e motora. Assim, quanto mais avançada a idade cronológica, maior vulnerabilidade para comprometimento da capacidade funcional ${ }^{22}$. Diante disso, é preciso refletir que a população idosa que era dependente nas atividades básicas foi composta por pessoas idosas acima de 70 anos.

O presente estudo identificou uma correlação positiva moderada com o MEEM, ou seja, quanto maior o nível de atividade, maior é a preservação cognitiva. A realização de atividades de forma rotineira tende a agir como fator protetivo à déficit cognitivo ${ }^{18}$.

Todavia, houve correlação entre AIVD, ABVD e MEEM, indicando uma dependência entre si de forma inversamente proporcional. Isso significa, em ambas as análises, que quanto menor a capacidade cognitiva, maior será o nível de dependência. Uma possibilidade para isso seria o deterioramento das capacidades funcionais e cognitivas que sofre influência direta do comprometimento do sistema nervoso ${ }^{3}$.

Portanto, diante da transição do perfil populacional, salienta-se a importância de se pesquisar acerca da capacidade funcional das pessoas idosas e o comprometimento cognitivo. O presente estudo traz dados que acrescentam a literatura uma análise sobre as pessoas idosas institucionalizadas, possibilitando comparações mais atuais.

\section{Limitações do estudo}

O estudo apresenta algumas limitações, tais como a ausência de inserção na avaliação de doenças incapacitantes, que podem influenciar na perda funcional de pessoas idosas e apresentação do déficit cognitivo. Além disso, por se tratar de um estudo transversal, os dados foram analisados em momento específico, assim, seria importante para o tema a realização de outros estudos que busquem realizar uma avaliação de causalidade.

\section{CONCLUSÃO}

Identificou-se que existe uma relação entre o déficit cognitivo e o nível de capacidade funcional de pessoas idosas institucionalizadas, de maneira que o déficit é menor na pessoa idosa que é mais independente e realiza mais atividades. Além disso, também foi possível identificar alguns fatores associados com o déficit: pertencer ao sexo masculino, apresentar uma menor idade, ser alfabetizado, nunca ter tido ou não têm mais a presença de um companheiro e não ter filhos. 
Esse estudo atesta sua relevância ao analisar dados que demonstram as condições funcionais e cognitivas de pessoas idosas institucionalizadas. Tais resultados ressaltam a importância do estímulo, por meio de cuidadores e enfermeiros, a avaliação das atividades funcionais e cognitivas para subsidiar o planejamento de medidas a serem adotadas para prevenção de agravos. Ademais, os achados deste estudo fortalecem a literatura e incentivam a realização de outros estudos.

\section{REFERENCES}

1. Cabral JF, Silva AMC, Mattos IE, Neves ÁQ, Luz LL, Ferreira DB, et al. Vulnerability and associated factors among older people using the family health strategy. Cienc. Saude Colet. [Internet] 2019 [cited 2021 Jan 12]; 24(9):3227-36. DOI: https://doi.org/10.1590/1413-81232018249.22962017.

2. Pereira XD, Araújo FL, Leite TI, Araújo FA, Bonfada D, Lucena EE. Prevalence and factors associated with cognitive impairments in the elderly of charity asylums: a descriptive study. Rev. Bras. Geriatr. Gerontol. [Internet]. 2020 [cited 2021 Oct 19]; 23(2):3757-65. DOI: https://doi.org/10.1590/1981-22562020023.200012.

3. Melo BRS, Diniz MAA, Casemiro FG, Figueiredo LC, Santos-Orlandi AA, Haas VJ, et al. Cognitive and functional assessment about elderly people users of health public service. Esc Anna Nery [Internet]. 2017 [cited 2020 Oct 13]; 21(4):1-8. DOI: https://doi.org/10.1590/2177-9465-ean-2016-0388.

4. Oliveira LB, Rodrigues IV, Boágua JS, Gomes EP. Suicide in old age: risk and protective factors. Brazilian Journal of Health Review [Internet]. 2021 [citado 2021 Oct 19]; 4(2):8337-49. DOI: https://doi.org/10.34119/bjhrv4n2-358.

5. Sposito G, Neri AL, Yassuda MS. Advanced Activities of Daily Living (AADLs) and cognitive performance in community-dwelling elderly persons: Data from the FIBRA Study - UNICAMP. Rev. Bras. Geriatr. Gerontol. [Internet]. 2016 [cited 2021 Feb 3]; 19(1):7-20. DOI: https://doi.org/10.1590/1809-9823.2016.15044.

6. Nazario MPS, Silva VHT, Martinho ACDO, Bergamim JSSP. Cognitive deficit in hospitalized elderly according to mini mental state examination (MMSE): narrative review. J. Heal. Sci. (Online) [Internet]. 2018 [cited 2020 Dec 13]; 20(2): 131. DOI: https://doi.org/10.17921/2447-8938.2018v20n2p131-134.

7. Barbosa LDM, Noronha K, Camargos MCS, Machado CJ. Social integration profiles among non-frail elderly institutionalized individuals in Natal, State of Rio Grande do Norte, Brazil. Cienc. Saude Colet. [Internet] 2020 [cited 2021 Oct 20]; 25(6): 2017 30. DOI: https://doi.org/10.1590/1413-81232020256.19652018.

8. Lourenço RA, Veras RP, Ribeiro PCC. Test-retest reliability of the Mini-Mental State Examination in an elderly population attended in a primary health care setting. Rev. Bras. Geriatr. Gerontol. [Internet]. 2019 [cited 2021 Oct 20]; 11:7-16. DOI: https://doi.org/10.1590/1809-9823.2008.11012.

9. Araújo GKND, Souto RQ, Alves FAP, Sousa RCRD, Ceballos AGDCD, Santos RDC, et al. Functional capacity and associated factors in the elderly: a population study. Acta Paul. Enferm. [Internet]. 2019 [cited 2021 Oct 20]; 32:312-18. DOI: https://doi.org/10.1590/1982-0194201900043.

10. Mendes SO, Ponte AS, Palma KAXA, Silva CGL, Delboni MCC. Validity and reliability of the Adapted Katz Index Scale. Research, Society and Development [Internet]. 2020 [cited 2021 Oct 20]; 9(4): 4. DOI: https://doi.org/10.33448/rsd-v9i4.2630.

11. Farías-Antúnez S, Lima NP, Bierhals IO, Gomes AP, Vieira LS, Tomasi E. Disability related to basic and instrumental activities of daily living: a population-based study with elderly in Pelotas, Rio Grande do Sul, 2014. Epidemiol. Serv. Saúde [Internet]. 2018 [cited 2021 Oct 20]; 27(2):e2017290. DOI: https://doi.org/10.5123/S1679-49742018000200005.

12. Oliveira A, Nossa $P$, Mota-Pinto A. Assessing functional capacity and factors determining functional decline in the elderly: $A$ cross-sectional study. Acta Med. Port. [Internet]. 2019 [cited 2021 Jan 7]; 32(10):654-60. DOI: https://doi.org/10.20344/amp.11974.

13. Araújo Neto AH, Patrício ACFA, Ferreira MAM, Rodrigues BFL, Santos TD dos, Rodrigues TD de B, et al. Falls in institutionalized older adults: risks, consequences and antecedents. Rev Bras Enferm [Internet]. 2017 [cited 2021 Jan 10]; 40(4):752-8. DOI: https://doi.org/10.1590/0034-7167-2017-0107.

14. Güths JFS, Jacob MHVM, Santos AMPV, Arossi GA, Béria JU. Sociodemographic profile, family aspects, perception of health, functional capacity and depression in institutionalized elderly persons from the north coastal region of Rio Grande do Sul, Brazil. Rev. Bras. Geriatr. Gerontol. [Internet]. 2017 [cited 2021 Jan 13]; 20(2):175-85. DOI: https://doi.org/10.1590/198122562017020.160058

15. Júnior FBA, Machado ITJ, Santos-Orlandi AA, Pergola-Marconato AM, Pavarini SCI, Zazzetta MS. Frailty, profile and cognition of elderly residents in a highly socially vulnerability area. Cienc. Saude Colet. [Internet]. 2019 [cited 2021 Jan 13]; 24(8):3047-56 DOI: https://doi.org/10.1590/1413-81232018248.26412017.

16. Santos CS, Bessa TA, Xavier AJ. Factors associated with dementia in elderly. Cienc. Saude Colet. [Internet]. 2020 [cited 2021 Apr 13]; 5(2):603-11. DOI: https://doi.org/10.1590/1413-81232020252.02042018.

17. Sousa NFS, Lima MG, Cesar CLG, Barros MBA. Active aging: Prevalence and gender and age differences in a population-based study. Cad. Saude Publica [Internet]. 2018 [cited 2021 Jan 15]; 34(11):1-16. DOI: https://doi.org/10.1590/0102-311X00173317.

18. Oliveira DV, Oliveira VB, Caruzo GA, Ferreira ÁG, Nascimento Júnior JRA, Cunha PM, et al. The level of physical activity as an intervening factor in the cognitive state of primary care older adults. Cienc. Saude Colet. [Internet]. 2019 [cited 2021 Jan 15 ]; 24(11):4163-70. DOI: https://doi.org/10.1590/1413-812320182411.29762017.

19. Brandão BMLS, Silva AMB, Souto RQ, Alves FAP, Araújo GKN, Jardim VCFS, et al. Cognition and quality of life relationship among the elderly community: a cross-sectional study. Rev. Bras. Enferm. [Internet]. 2020 [cited 2021 Jan 15]; 73(3):1-7. DOI: https://doi.org/10.1590/0034-7167-2019-0030. 
20. Fernandes JS, Costa BHR, Andrade MS. Social representations about family of the elderly. Ciências Psicológicas [Internet]. 2017 [cited 2021 Jan 16]; 11(1):41-8. DOI: https://doi.org/10.22235/cp.v11i2.1345.

21. Pinto AH, Lange C, Pastore CA, de Llano PMP, Castro DP, Santos F. Functional capacity to perform activities of daily living among older persons living in rural areas registered in the Family Health Strategy. Cienc Saude Cole [Internet]. 2016 [cited 2021 Feb 13]; 21(11):3545-55. DOI: https://doi.org/10.1590/1413-812320152111.22182015.

22. Nunes JD, Saes MO, Nunes BP, Siqueira FCV, Soares DC, Fassa MEG, et al. Functional disability indicators and associated factors in the elderly: a population-based study in Bagé, Rio Grande do Sul, Brazil. Epidemiol. Serv. Saúde [Internet]. 2017 [cited 2021 Feb 13]; 26(2):295-304. DOI: https://doi.org/10.5123/s1679-49742017000200007. 